\title{
Investigación
}

\section{Computación \\ en el borde y \\ en la niebla}

DOI: 10.29236/sistemas.n156a4

Tendencias e inmersión

\section{Resumen}

La Computación en el borde y en la niebla contempla estrategias para extender el paradigma de Computación en la nube, adoptado en forma amplia hoy por hoy, en el ámbito organizacional e incluso personal. Ha adquirido importancia creciente en los últimos años como una alternativa en modelos centralizados, que ofrece respuestas a retos latentes en las aplicaciones del entorno de Internet de las Cosas.

A la luz de este panorama tecnológico, la Asociación Colombiana de Ingenieros de Sistemas (Acis), realizó una encuesta en el contexto nacional, dirigida a ingenieros de sistemas y computación, con el fin de conocer su opinión, conocimiento y grado de aplicación de estas dos tecnologías, "computación en el borde" y "computación en la niebla", cuyos resultados y reflexiones, son presentadas en este artículo.

Palabras clave: Computación en el Borde (Edge Computing), Computación en la Niebla (Fog Computing), Internet de las Cosas (IoT), Seguridad. 


\section{Introducción}

El escenario tecnológico definido por Internet de las Cosas y por aplicaciones que demandan respuesta en tiempo real, plantea al sector empresarial y a los desarrolladores en el campo de la tecnología de información y las comunicaciones, desafíos de carácter funcional y tecnológico. La Computación en la niebla y la Computación en el borde constituyen una alternativa para hacer frente a algunos de esos desafíos como la movilidad, la respuesta en tiempo real y el uso eficiente de recursos.

La primera ofrece un mejor procesamiento de los datos para las aplicaciones basadas en la nube, el cual realiza más cerca de la fuente de los mismos, en el borde de la red. Gracias a esta característica se alcanzan beneficios como: menor latencia entre la aplicación cliente y el servicio en la nube; acercamiento del usuario y dispositivos a los contenidos con un uso más eficiente de los recursos de red y habilitación de la infraestructura de red futura.

Por su parte, la Computación en la Niebla enfatiza en el procesamiento en la infraestructura local de red, más que en los dispositivos y extiende los recursos de computación hacia el borde de la red, en un modelo distribuido. Así, se dispone en el borde de los servicios de procesamiento, almacenamiento y moni- toreo provistos por el enfoque tradicional en la nube, por medio de instancias denominadas "fog nodes", las cuales realizan un procesamiento previo de los datos a la nube, en los dispositivos loT ubicados en el borde, con beneficios potenciales como: menor latencia entre los dispositivos de usuario final y los nodos en la niebla; soporte a requerimientos de movilidad; habilitación de la ubicuidad de los servicios de computación y viabilidad de interacciones en tiempo real.

No obstante, la computación en la niebla y en el borde, tiene retos vislumbrados en el escenario tecnológico actual, asociados con los costos adicionales de hardware para el procesamiento local, las fallas de seguridad y confiabilidad de los dispositivos o de la red, o bien, el mantenimiento y control, retos que la computación centralizada en la nube no incluye.

A la luz de este panorama tecnológico, Acis realizó la encuesta Computación en el borde y en la niebla, tendencias e inmersión, dirigida a ingenieros de sistemas y computación, cuyos resultados reflejan la opinión, conocimiento y grado de aplicación de estas dos tecnologías, analizados en este documento.

La encuesta fue realizada durante los meses de julio y agosto de 2020 , incluyó 10 preguntas orienta- 
das a detectar el grado de conocimiento y apropiación de Fog y Edge Computing por parte de los ingenieros las organizaciones en donde se desarrollan profesionalmente. Los resultados muestran un universo de 86 encuestas diligenciadas al $100 \%$ y un tiempo de respuesta promedio de seis minutos y ocho segundos. Para la aplicación de esta encuesta se utilizó la herramienta abierta SurveyMonkey.

\section{Resultados de la encuesta}

En este aparte se presentan los resultados de las 10 preguntas que componen la encuesta con el correspondiente análisis individual y global, en los cuales se evidencia no solo el grado de apropiación, sino el impacto actual y potencial asociado a las tecnologías Fog $y$ Edge computing y que puede servir de base para fomentar su difusión y uso en proyectos de ingeniería y desarrollo asociados con TICs y particularmente en el entorno demarcado por Internet de las Cosas, que sin duda permea espacios laborales y personales de la vida, en el siglo XXI.

\section{Pregunta 1: Conocimiento y comprensión de Fog y Edge Computing}

Es fundamental estimar el grado de conocimiento de estos dos conceptos y las tecnologías asociadas en

Figura 1. En su práctica profesional, los conceptos: "computación en el borde" y "computación en la niebla"

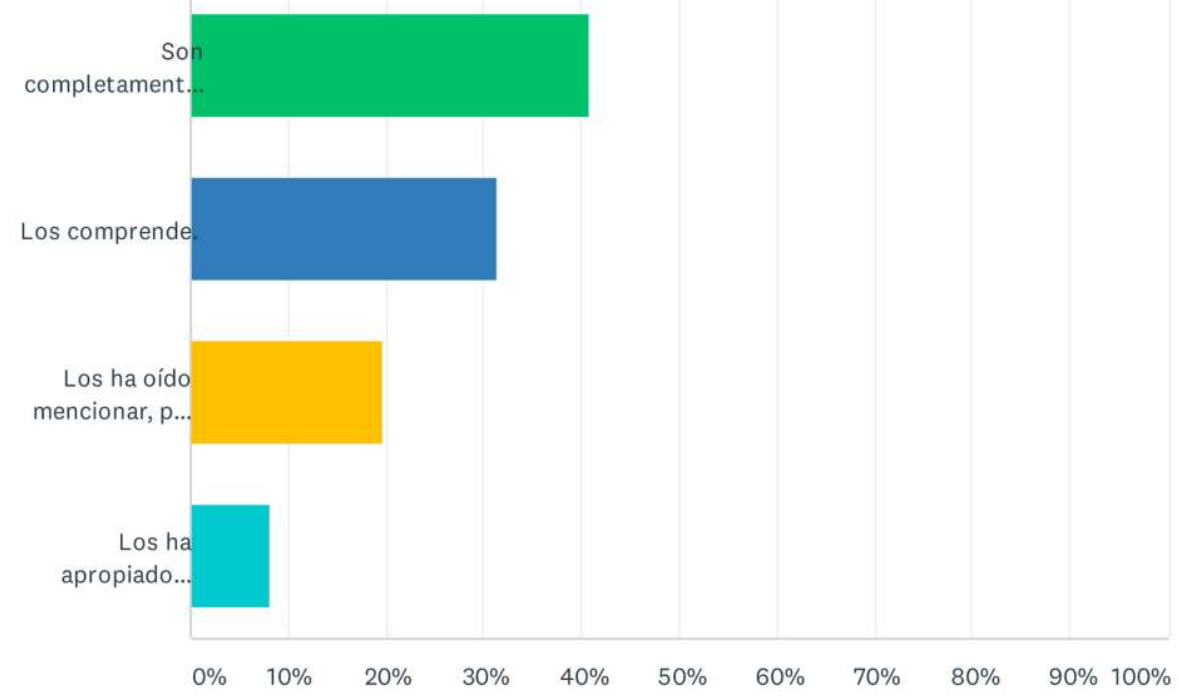


la práctica profesional de ingenieros y desarrolladores e identificar en qué medida se están aplicando.

En la Figura 1, los resultados revelan un panorama incipiente de conocimiento, en un $60.47 \%$ de los encuestados, para quienes son conceptos totalmente nuevos o que han oído mencionar, pero no comprenden. No obstante, un $31.4 \%$ de los encuestados manifiesta comprenderlos, porcentaje relevante, que da cuenta de una dinámica en curso, que potencia un índice de aplicación en un futuro próximo y deja entrever un posible nicho para el fomento de iniciativas que apliquen Fog y Edge computing.

Sin embargo, es necesario reconocer según estos resultados, que estos conceptos son nuevos para la mayoría de los encuestados, y la apropiación de estas tecnologías puede ser baja en el momento actual del país.

Pregunta 2: Uso de servicios de cómputo Fog / Edge

Computing

En ecosistemas como el definido por Internet de las Cosas, caracterizados por el tiempo real, la necesidad de una baja latencia, aplicaciones distribuidas geográficamente y dispositivos capaces de procesar in situ, resulta más que ideal acercar los servicios al usuario de aplicaciones.

Tales opciones contemplan servicios de almacenamiento, infraestructura, y procesamiento de los datos, sin necesidad de ir hasta la nube, la cual aparece "lejana" para muchas aplicaciones loT.

Por otra parte, en los modelos actuales de negocio, el acceso a ser-

Figura 2. ¿Conoce y/o ha utilizado algún servicio de cómputo en el borde (edge computing) o en la niebla (fog computing)?

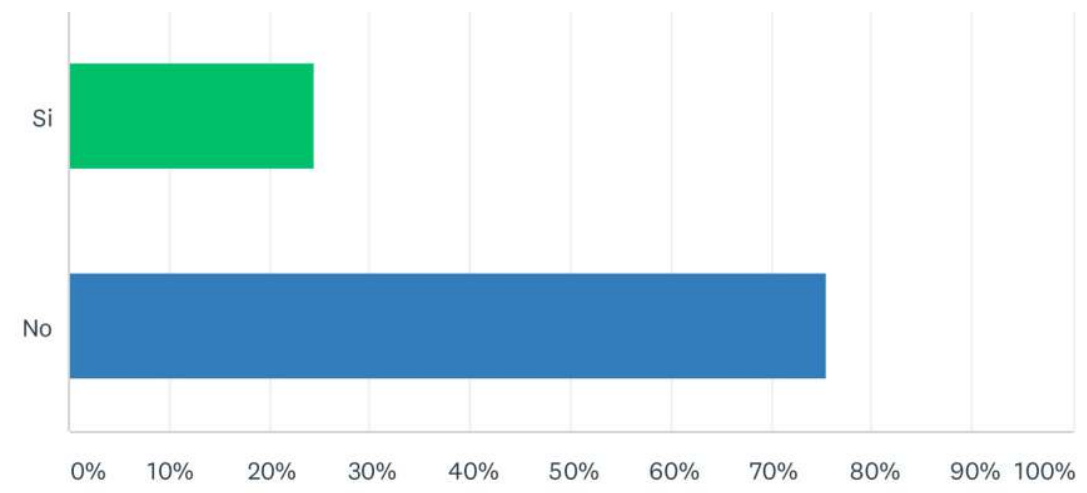


vicios es una alternativa muy atractiva que permite a las empresas crecer con sistemas escalables, cuyo costo de actualización, infraestructura, costos de actualización e incluso formación de expertos en TI, se traslada a los proveedores de servicio, dando paso con esto, incluso a una mejor planeación y gestión de gastos.

A pesar de esta tendencia global hacia el uso de servicios y de las ventajas que ofrecen la computación en la niebla y en el borde especialmente en aplicaciones de internet de las cosas, los resultados obtenidos en la encuesta sobre este particular, señalan una proporción de 3 a 1 (Ver Figura 2), en la cantidad de personas o empresas que no conocen ni han usado algún servicio de cómputo fog / edge y quienes sí los conocen. Esto de manera decidida, sugiere la necesidad de adelantar más iniciativas de difusión sobre estas tendencias y alternativas tecnológicas, como la que persigue Acis en esta edición de la revista Sistemas y con la encuesta misma, objeto de análisis. El mayor conocimiento de estos conceptos, de sus tecnologías asociadas y análisis de casos de uso, serán esenciales, para la adopción de este tipo de servicios y modelos de arquitecturas.

\section{Pregunta 3: Necesidad de la} computación en el borde o en la niebla.

La confianza de las empresas y profesionales de $\mathrm{TI}$ juega un rol fundamental en el proceso de evolución de tecnologías como estas, objeto de la encuesta realizada, proceso que será exitoso, en la medida en que efectivamente se apro-

Figura 3. En su opinión la computación en el borde o en la niebla es:

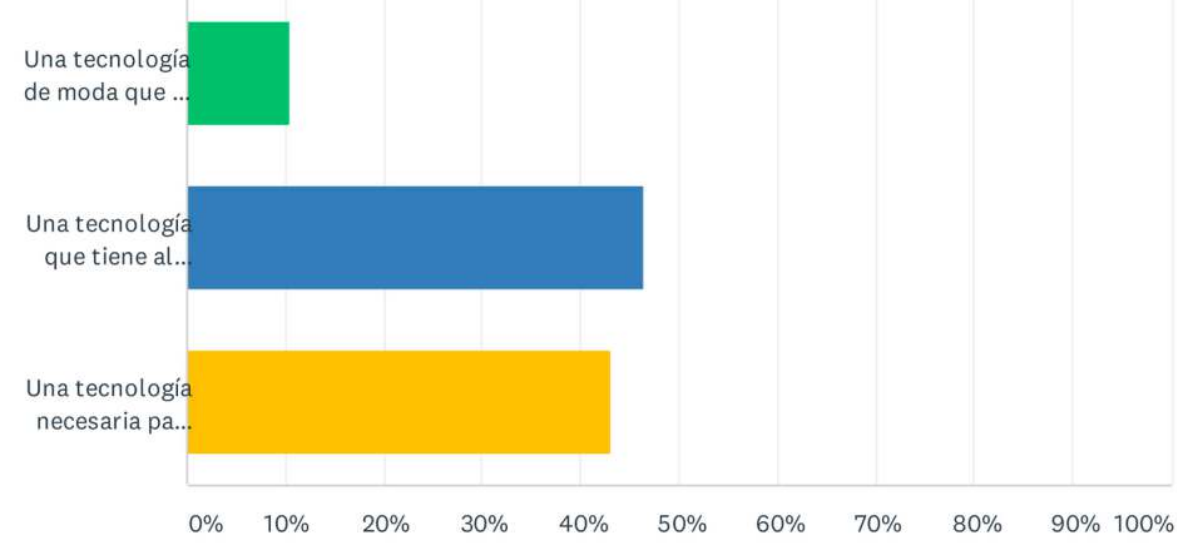


pien y apliquen; en ese orden de ideas, los resultados obtenidos para esta pregunta son bastante alentadores, de cara a las posibilidades que tienen estas tecnologías. La Figura 3 muestra un escenario en el cual sólo un $10 \%$ no espera el uso masivo de estas tecnologías mientras que la gran mayoría (un $89.52 \%$ ), percibe en ellas potencialidad en relación con la ventaja competitiva para sus empresas y casi la mitad de ellos, le otorga un carácter esencial para alcanzar tal ventaja competitiva.

La competitividad, junto con los costos, el acceso distribuido, la escalabilidad y disponibilidad, entre otros, son factores a tener en cuenta en la elección de una alternativa tecnológica, en el momento actual y estos factores obtienen una buena valoración en el caso de la Com- putación en el borde y en la niebla. Es posible que las respuestas obtenidas obedezcan a este tipo de análisis, lo que justifica ese $89.52 \%$ de respuestas que asocian competitividad con estas tecnologías.

\section{Pregunta 4. Seguridad en el} borde y en la niebla vs la nube En un escenario que alienta la generación continua de datos en forma masiva, el almacenamiento y procesamiento en servidores externos, algunas veces públicos y la transferencia de datos empresariales y personales fluyendo por redes externas, la seguridad y privacidad de los datos, en muchos casos sensibles, debe ser un criterio a considerar.

Si bien la primera disyuntiva de cara a la seguridad es elegir un proveedor de servicios como infraes-

Figura 4. Considera que una aplicación que utiliza un componente de cómputo en el borde o en la niebla es:

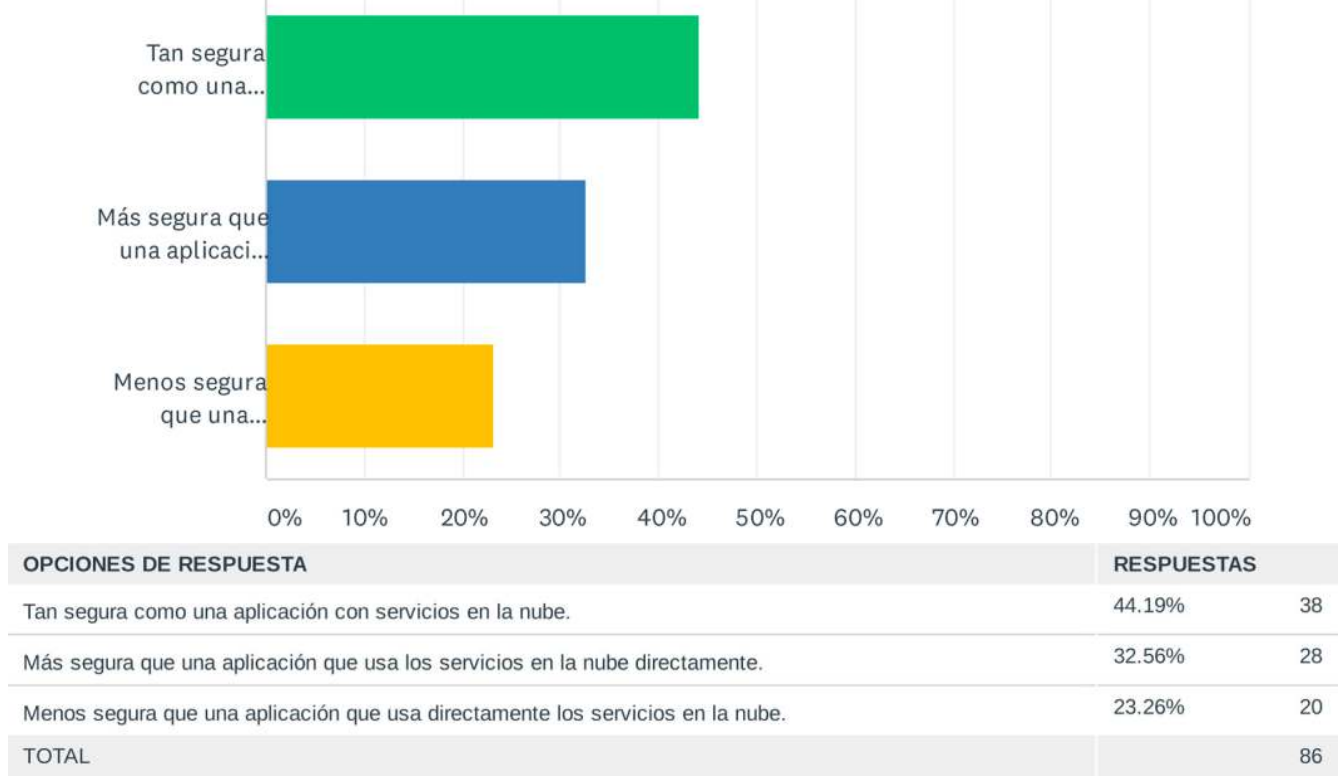


tructura, plataforma o software interno o externo, esta pregunta de la encuesta abordaba una disyuntiva más: si al ser externo, se percibe mayor seguridad en la nube, o en la niebla o el borde. La seguridad y también la privacidad de la información es un asunto de interés ante la adopción de nuevas tecnologías, es una constante.

Sin embargo, los resultados de la encuesta parecen reflejar una baja preocupación; la mayoría, más del $76 \%$, considera que la computación en el borde es tanto o más segura que la computación en la nube y probablemente este resultado evidencia desconocimiento de los riesgos por parte del público general, más que confianza objetiva en la tecnología. (Ver Figura 4).
Pregunta 5: Eficiencia en el procesamiento de los datos en el borde o en la niebla

Para dar respuesta a los retos de velocidad, variedad y volumen de los datos loT, Cisco ${ }^{1}$ plantea requerimientos asociados a la latencia, el ancho de banda de la red, la seguridad, la integridad y disponibilidad de la infraestructura y los datos, la gestión de datos distribuidos geográficamente y ubicar los datos en el mejor lugar para su procesamiento. Tales requerimientos no son satisfechos completamente en las arquitecturas tradicionales de computación en nube. Propone, por tanto, procesar la mayoría de

CISCO, 2015 - Fog Computing and the Internet of Things: Extend the Cloud to Where the Things Are

Figura 5. En un escenario loT de "cosas" autónomas que capturan información, las soluciones tecnológicas deben privilegiar el procesamiento eficiente y en tiempo real de los datos. Considera usted que la adopción de la computación en el borde y la niebla en las empresas:

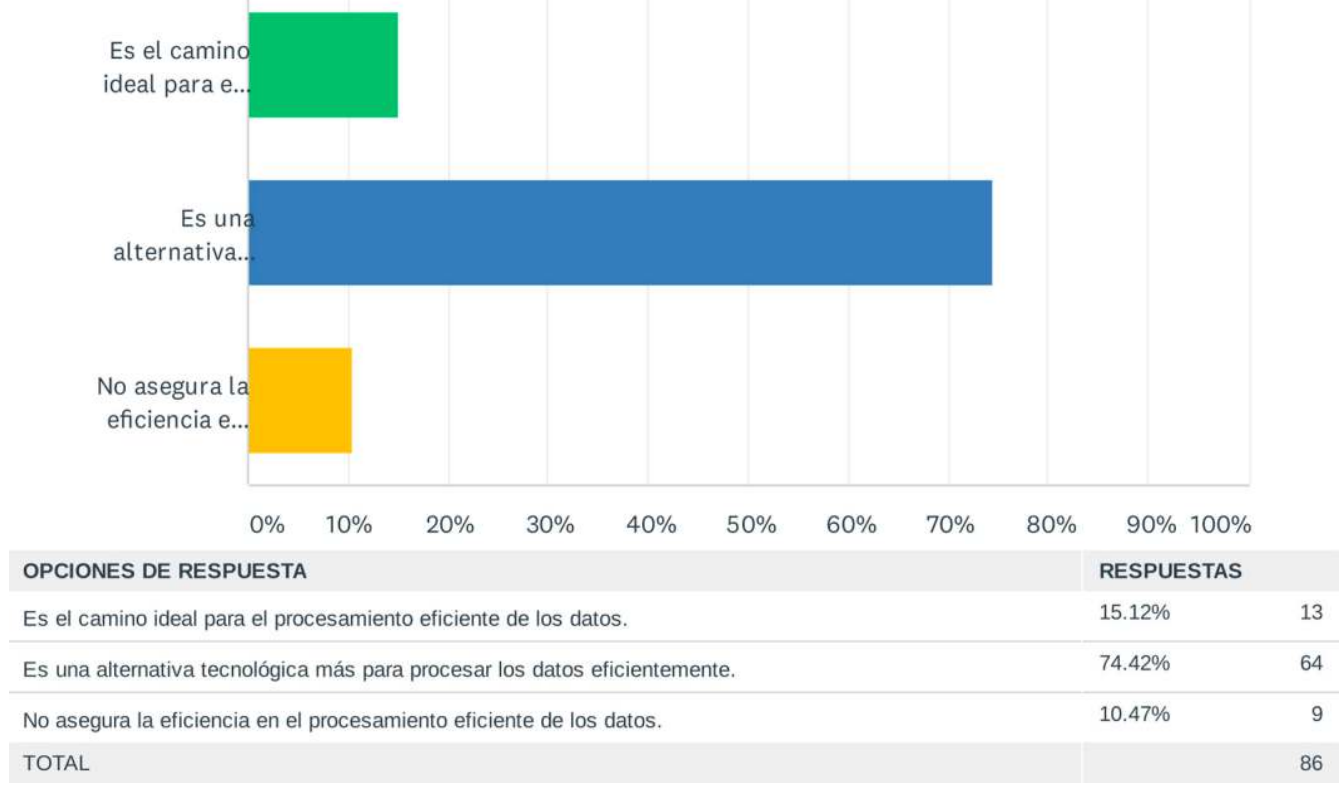


los datos loT cerca de los dispositivos que producen y actúan sobre los mismos, es decir, mediante la arquitectura de computación en la niebla.

Estas posibles ventajas que otorga el procesamiento en la niebla o en el borde, parecen reconocerse según los resultados de la encuesta que advierten confianza en estas arquitecturas computacionales, otorgando un $74.42 \%$ como una alternativa tecnológica más para el procesamiento de los datos, y un $15.12 \%$, lo visualiza como la alternativa ideal. Así, solo un $10.47 \%$ manifiesta escepticismo frente a su eficacia en el procesamiento de los datos. Una vez más, se vislumbra una oportunidad para la apropia- ción futura de estas tecnologías, con un $89.54 \%$ de reconocimiento de su eficacia (Ver Figura 5).

\section{Pregunta 6: Percepción de las} empresas acerca de la adopción de la tecnología de Computación en el borde

El "hype cycle" o "ciclo de sobreexpectación" creado por la consultora tecnológica Gartner constituye una herramienta muy reconocida para comprender el proceso de introducción de las innovaciones preferencialmente tecnológicas, en el mundo empresarial y de negocios.

Su pronóstico sobre la inmersión de la Computación en el Borde, responde positivamente a la expectativa que suscita el uso de la com-

Figura 6. La consultora Gartner estima que en 2025 el 75\% de los datos empresariales se procesarán en el borde, en comparación con el 10\% actual. Este pronóstico plantea una alta inmersión en el entorno empresarial de este enfoque tecnológico. En su caso, los directivos de su empresa:

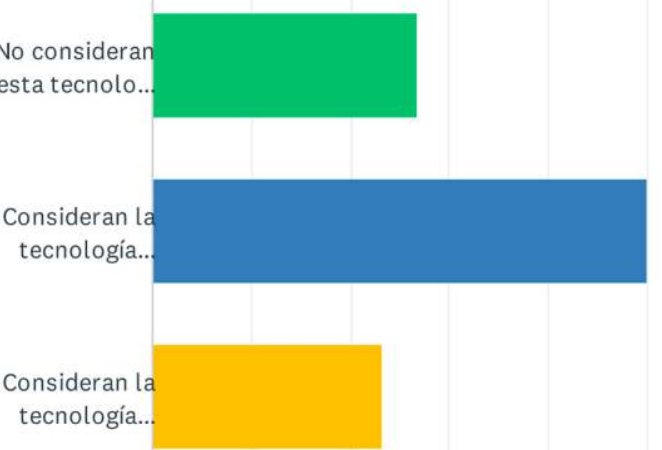

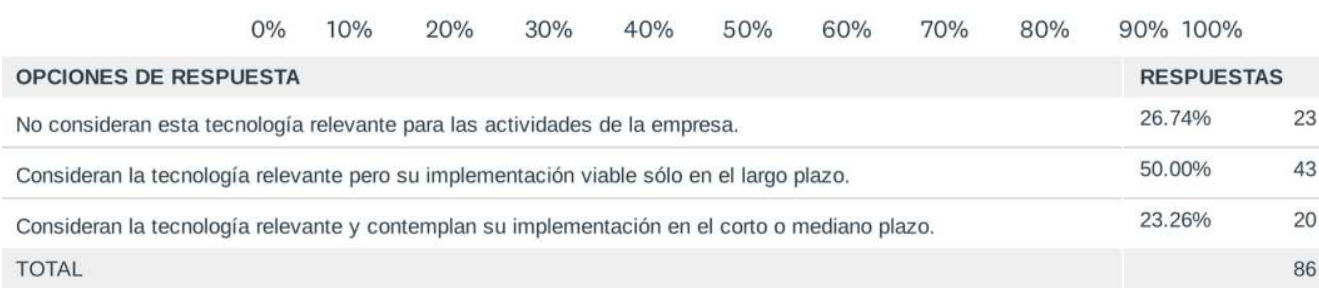


putación en la nube, en ciertas aplicaciones, primordialmente en lo relacionado con la latencia, el procesamiento distribuido y la seguridad de los datos.

Los resultados permiten identificar el reconocimiento de la relevancia de la Computación en el Borde, por parte de las empresas y expertos de $\mathrm{TI}$, con un $73.26 \%$ en total, visibles en la Figura 6; no obstante, casi dos terceras partes de ellos, la perciben viable sólo en el largo plazo. Esto revela una demanda por mayor formación de expertos en estas tecnologías, y la necesidad de contar con más casos de uso significativos que permitan evidenciar el apalancamiento de las empresas a partir del uso de estas tecnologías.
Debe mencionarse también ese $26.74 \%$ que no considera estas tecnologías relevantes para su empresa, explicable en la idea de que no existe nada posible en el borde, que no se pueda realizar también en la nube. Quizás la respuesta sea obvia para empresas de sectores como la manufactura, en la que una latencia de milisegundos para tomar una decisión puede ser determinante, pero es cierto también que hay decisiones que soportan los tiempos de respuesta que ofrece la nube.

Pregunta 7: Proceso de adopción de la Computación en el Borde y en la Niebla en las empresas

Cuando en 1979 Alvin Toffler anunció en su libro², la inminencia de la

Figura 7. Considerando el efecto de la adopción de la computación en el borde y en la niebla sobre la arquitectura de las aplicaciones, las empresas que tienen aplicaciones basadas en la nube deberían:

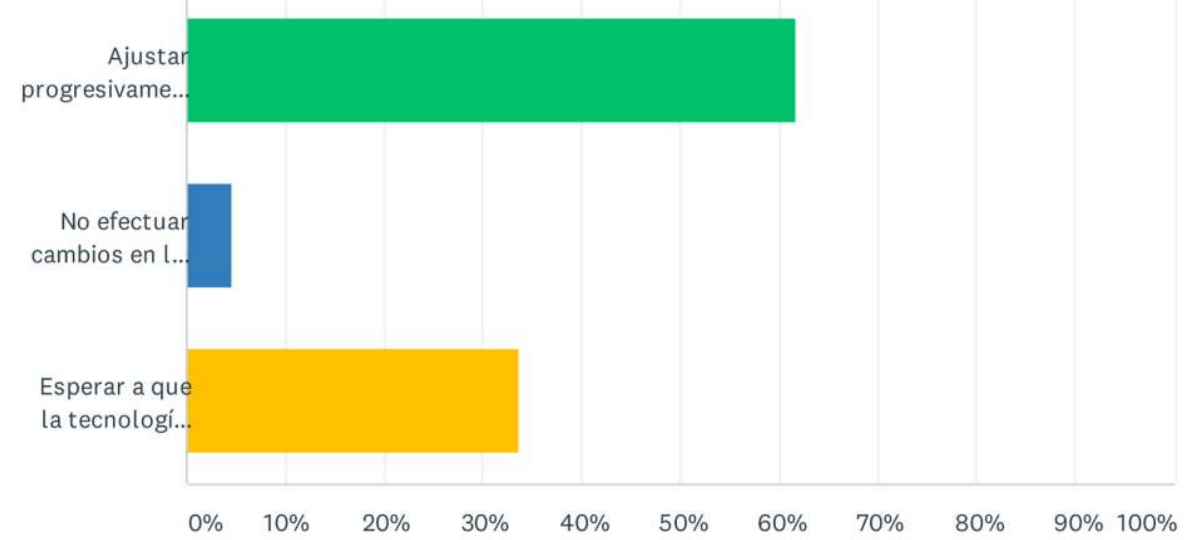


tercera ola con cambios significativos en diversos ámbitos de la vida humana, basado en una revolución de las TICs, con un sistema de producción basado en la información, medios de comunicación globalizados, interactivos y colaborativos, probablemente contribuyó a prepararnos para el cambio, pero quizás no, para la velocidad y contundencia con la sobrevendrían las siguientes olas.

El final del siglo $X X$ y las dos primeras décadas del XXI, han enfrentado a los tomadores de decisiones en las organizaciones a cambios continuos y a tecnologías disruptivas que desafiaron sus sistemas, procesos y paradigmas.

Las respuestas de los encuestados frente al proceso adecuado de sus empresas para adoptar la Computación en el Borde y en la Niebla revelan una posición de cautela, representada en un $66.63 \%$ que considera que se debe llevar a cabo un ajuste progresivo de las aplicaciones, más un $33.72 \%$ que preferirían esperar a que estas tecnologías tengan un uso más generalizado antes de hacer cambios (Ver Figura 7).

De nuevo se requieren más casos de uso que puedan evidenciar las ventajas reales de estas tecnolo-

TOFFLER, Alvin. La Tercera Ola, 1993. - Plaza \& Janes gías y su potencial para incrementar la competitividad de las empresas.

\section{Pregunta 8: Compromiso con} la seguridad de los datos en los servicios en el borde, debido al alto número de dispositivos (IoT)

Con una arquitectura IoT, los servicios en el borde suponen el procesamiento de los datos, incluso en los mismos dispositivos, y exige por tanto garantizar su inteligencia y autonomía, además de dotarlos de microcontroladores para dicho procesamiento y para poder "hablar" con otros dispositivos locales o externos.

Este escenario, sin embargo, implica un esfuerzo por parte de los directivos para proteger los sensores y dispositivos ante fallos o amenazas o intrusiones que pueden poner en riesgo no solo los datos, en muchos casos datos sensibles, sino también la correcta ejecución del sistema.

Es por ello comprensible la posición de los encuestados, de los cuales un $72.09 \%$ (Ver Figura 8) ve comprometida la seguridad de los datos al utilizar servicios en el borde.

No obstante, un $27 \%$ no encuentra este tipo de problemas y los análisis aquí pueden variar desde un verdadero desconocimiento de dichos riesgos o amenazas o bien, con una visión más optimista, a par- 
tir de experiencias exitosas con el uso de servicios en la nube, la niebla o incluso en el borde mismo.

\section{Pregunta 9: Riesgos de} seguridad más relevantes identificados por los servicios en el borde Los riesgos de seguridad en el uso de servicios en el borde que más preocupan a los ingenieros y expertos de $\mathrm{TI}$, están claramente asociados con la confidencialidad de los datos. Un $78.82 \%$ de los encuestados, prioriza este aspecto, aunque un $44 \%$ también encuentra amenazas a la seguridad por pérdida de integridad y un $37.65 \%$ por pérdida de disponibilidad, como se observa en la Figura 9. En este sentido, a pesar de que muchos manifestaron en respuestas previas el desconocimiento y otros la falta de com- prensión de estas tecnologías, existe un acierto al visualizar amenazas en estos 3 aspectos, sobre los cuales la mayor inmersión en uso de servicios en el borde, deberá dar respuestas a directivos y líderes de TI que eleven la confianza de los usuarios y permitan que más usuarios acojan esta nueva ola tecnológica.

\section{Pregunta 10: Percepción sobre} apalancamiento de la Computación en el Borde y en la Niebla, para la adaptación de las empresas al contexto digital Siendo ésta una pregunta abierta, pueden resumirse las respuestas en una mayoría que la encuentra atractiva y le concede posibilidades para apoyar la resiliencia de las operaciones de las empresas, pero con salvedades garantizar la segu-

Figura 8. ¿Considera usted que la seguridad de los datos se verá comprometida al utilizar servicios de cómputo en el borde, teniendo en cuenta el alto grado de proliferación de dispositivos conectados en las empresas y en los hogares (IOT)?

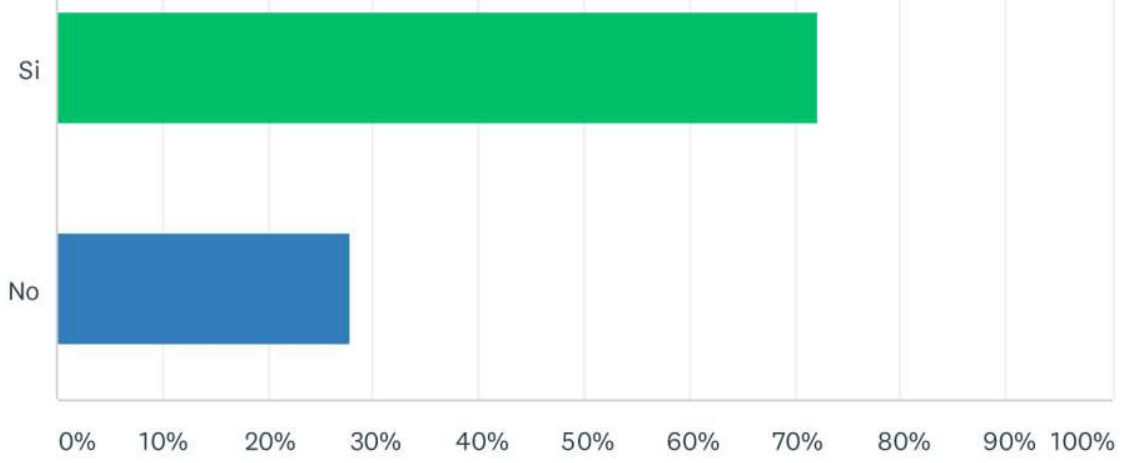


ridad de los datos, o la comprensión del "tiempo real" que no siempre es real, sino cercano al real, dependiendo del tipo de sistema o empresa.

Sin embargo, algunos la encuentran muy atractiva, considerando las circunstancias que enfrentó el planeta en el presente año, con exigencias de conectividad y comunicación efectiva en las corporaciones, en entornos netamente digitales.

Cabe señalar un grupo menor de respuestas que se acogen a modelos más conservadores, los cuales suponen gestionar los datos a nivel local o bien el uso de la nube, con servidores que respondan a sus servicios de computación, sin poner en riesgo la seguridad de sus datos.

Hay bastante franqueza en algunas respuestas sobre el desconocimiento de este impacto, manifiesto en forma explícita o por la omisión en la respuesta.

\section{Conclusiones}

Esta investigación permite formular algunas conclusiones, relacionadas a continuación:

- Se requieren mayores esfuerzos para que los directivos y expertos en TI conozcan y entiendan estas tecnologías, idealmente con la ilustración a través de casos de uso significativos, que in-

Figura 9. Si la respuesta es afirmativa, qué riesgos de seguridad identifica como los más relevantes: (señale todos aquellos que apliquen).

Pérdida de confidencial..

Pérdida de disponibilidad

Pérdida de

integridad

$$
\text { d }
$$

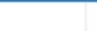


crementen su confianza y evidencien su potencial en cuanto a procesamiento eficiente y ventajas competitivas para las organizaciones.

- Si bien las alternativas de procesamiento en el borde en un entorno loT, ofrecen respuestas en cuanto a la velocidad de respuesta, la seguridad de los datos y la eficiencia del procesamiento, persiste escepticismo en los usuarios atinentes a la confiden- cialidad, la disponibilidad y la integridad de los datos.

- La velocidad de cambio de las tendencias tecnológicas, desafía los ciclos de evolución y maduración de los sistemas de información y plantea retos a directivos y tomadores de decisiones, con tecnologías disruptivas que permean los paradigmas organizacionales, con alternativas a veces no consolidadas o suficientemente probadas.

Libia Denisse Cangrejo Aljure Ingeniera de Sistemas, PhD en Ingeniería - Sistemas y Computación y Msc. en Geomática, de la Universidad Nacional de Colombia. Especialista en Teledetección, Cartografía y SIG de la Universidad Alcalá de Henares, España y especialista en SIG, de la Universidad Distrital Francisco José de Caldas. Desarrolló su tesis doctoral en el campo de Modelado Semántico de Contexto para el ámbito de Internet de las Cosas, con Linked Open Data. Docente de la Facultad de Ingeniería de la Universidad Nacional de Colombia. Ha participado y liderado proyectos de TI y Geoinformación en diversos campos, algunos de ellos de carácter social, como EI SIG de Gestión Local para Ciudad Bolívar, en la Corporación SUR, Georreferenciación y Reingeniería para la Gestión del Conflicto Local de la Candelaria, Diseño conceptual del SIG para la Mesa Regional de Planeación Bogotá-C/marca, PNUDR/UNAL y el Convenio UNAL/MinTIC, Computadores para Educar, entre otros. 